\title{
A Model of the Distribution of Wealth in Society
}

\begin{abstract}
H. Lavička, F. Slanina
A model of the distribution of wealth in society will be presented. The model is based on an agent-based Monte Carlo simulation where interaction (exchange of wealth) is allowed along the edges of a small-world network. The interaction is like inelastic scattering and it is characterized by two constants. Simulations of the model show that the distribution behaves as a power-law and it agrees with results of Pareto.
\end{abstract}

Keywords: Pareto's law, economics, scattering.

\section{Introduction}

Philosophers and scientists in the 19th century started to investigate many natural and social phenomena. In fact, the 19th century was a revolutionary era during which the first "natural law" of economics [1] - Pareto's law was observed. Pareto's law states that the high end of wealth distribution follows the power-law $P(w) \sim w^{-1-\alpha}$, where exponent $\alpha$ is stable for an investigated country in a given period of time.

Many scientists have questioned the validity of Pareto's law and they have made measurements of the distribution, but the main message still remains true - the higher end of wealth distribution behaves like the power law. Experiments in e.g. $[2,3,4,5]$ performed in the last few years, have shown the validity of Pareto's law. The functional form itself is not amazing but the stability of the law in time and space is remarkable. The value of exponent $\alpha$ varies slightly from one country to another and there are small fluctuations of exponent $\alpha$ in time, but Pareto's law has been found almost everywhere. Moreover, the validity of Pareto's law can be extended back to ancient Egypt, to the times of the Pharaohs [6].

This universality of the power-law tail is a surprising phenomenon, and it asks for an explanation. Recent studies [7, 8, $9,10,11,12,13,14,15,16,17,18,19,20]$ have investigated the multiplicative random process repelled from zero as a mathematical source of power-law distributions. However, there are a million ways to implement multiplicative random processes, and the most studied implementations are the generalized Lotka-Volterra equation $[10,11,12,13]$ and the analogy with directed polymers in random media [21, 22, 23]. In these methods, models are formed by a kinetic equation that describes the exchange of wealth in a society of agents and global redistribution which is analogous to repelling from zero in stochastic processes.

Empirical studies of the lower end of the distribution have shown exponential behavior [3, 24, 25] and this behavior has been interpreted as a conservation law for total wealth, which leads to the robust Boltzmann exponential distribution that is analogous to the energy distribution in a gas of elastically scattering molecules.

Similar studies in [26] with previous notes lead to the view of economic activities as a scattering process, where the agents are analogous to inelastic scattering particles [27, 28, 29, $30,31,32,33]$. Inelasticity is very important to explain the power-law tail of wealth distribution. The assumption that there is a total wealth increase on average is reasonable for economic reasons (e.g., rising GDP).

Inelastical scattering of particles has been investigated in the context of granular materials [34] and the Maxwell model and its inelastical variants, e.g., [35, 36]. These studies lead to the conclusion that a self-similar solution of kinetic equations exists. This solution is not stationary but assumes a time-independent form after rescaling the energy, and the tail of the scaling function is the power-law when certain conditions are used.

A theoretical investigation of inelastical scattering agents on a fully-connected network (mean field solution) is performed in [37] and power-law tails of wealth distribution were found for a large set of parameters $\varepsilon, \beta$ of the interaction. It is suggested in [37] that the theoretical solutions do not answer the problem of the robustness of exponent $\alpha$ in different societies and the answer could be given by a sociological ingredient in the model.

Recent investigations of networks, which has been reviewed in [38], show some remarkable phenomena, and models that agree with the basic experimental measurements have been introduced e.g. in [39, 40]. One possible enhancement of the model could be the use of networks where interaction is allowed only along the edges. This paper deals with simulations of the model in [37] on the Watts- Strogatz network [39].

\section{Definition of the model}

Let us imagine a society of $N$ agents, where each agent has only one variable which signs his/her wealth $\widetilde{w}_{i}$, $i \in\{1,2, \ldots, N\}$. Thus, the state of the system is described by $W=\left\{\widetilde{w}_{1}, \widetilde{w}_{2}, \ldots, \widetilde{w}_{N}\right\}$. The agents are able to interact and the interaction is essentially instantaneous. Of course, a real society is more complicated and many economic interactions can take place at the same time, pairwise, although some economic interactions can be taken as multilateral rather than bilateral in a real society, and positive, the interaction has a positive effect on the total wealth of the society of the agents. Thus, the interacting agents become, in sum, more wealthy after the interaction than at the beginning of the interaction.

When two agents $i$ and $j$ are chosen to interact, the dynamics of the wealth of agents $i$ and $j$ is governed by interactions that can be formalized as follows 
$\left(\begin{array}{r}\widetilde{w}_{i}(t+1) \\ \widetilde{w}_{j}(t+1)\end{array}\right)=\left(\begin{array}{rr}1+\varepsilon+\beta & \beta \\ \beta & 1+\varepsilon-\beta\end{array}\right)\left(\begin{array}{c}\widetilde{w}_{i}(t) \\ \widetilde{w}_{j}(t)\end{array}\right)$

and all other agents remain unchanged, so $\widetilde{w}_{k}(t+1)=\widetilde{w}_{k}(t)$ for $k \neq i$ and $k \neq j$ where $\varepsilon$ and $\beta$ are parameters of the model. $\beta \in(0,1)$ measures the strength of the exchange and $\varepsilon>0$ measures the one-step inow of wealth.

Interaction is allowed only along the edges of a network, which is represented by the graph $\Lambda=(\Gamma, E)$, where $\Gamma$ is the set of all nodes and $E$ is the set of all edges. Edge $e$ is a unordered pair $e=(i, j)$ connecting nodes $i$ and $j$. Each node $i$ from $i \in \Gamma$ has its neighborhood $\Gamma_{i} \in\{j \in \Gamma \mid(i, j) \in \Gamma\}$. Each agent $i$ is bound to its own node $i$ and the agent's neighborhood is $\Gamma_{i}$.

The network is generated by the Watts-Strogatz algorithm [39], which supports the network with basic features that have been found to describe human networks. A rewiring algorithm is applied to a totally ordered network, which means that each edge is rewired to a randomly chosen agent with probability $p$.

There are two possible ways to execute one Monte Carlo step, using

- an agent initiated model,

- an edge initiated model.

\subsection{Agent initiated model}

The updating mechanism of the Monte Carlo step is based on the choice of agents i.e., agent $i \in \Gamma$ is chosen with uniform distribution and a second agent $j$ is chosen with uniform distribution from his/her neighbors $\Gamma_{i}$. It can be argued that the edges of the graph are only dispositions that can be used by agents and pair agents that interact, are interested in collaboration, and the collaboration is useful for them.

\subsection{Edge initiated model}

This model is based on the choice of an edge $e=(i, j)$ with uniform distribution. The interacting agents are signed $i$ and $j$, the rule of interaction is symmetric to the exchange of $i$ for $j$, so there is no ambiguity. It can be argued that every connection in society is used with the same probability, and highly connected agents will interact very frequently.

\section{Interesting variables}

Measured wealth was normalized $w_{i}=\widetilde{w}_{i} / \bar{w}$. This means that there are $N$ units of wealth in the society after normalization, and they are distributed among the agents.

The first interesting variable is social tension, which measures differences in wealth

$T_{\sigma}=\frac{1}{\bar{w}}\left(\frac{1}{|E|} \sum_{i \in E} \frac{1}{\left|\Gamma_{i}\right|} \sum_{j \in \Gamma_{i}}\left|w_{i}-w_{j}\right|^{\sigma}\right)^{\frac{1}{\sigma}}$

where $\bar{w}=\frac{1}{N} \sum_{i \in E} w_{i}$ so it is the average. $\sigma \in(0,1)$ is a parameter set up to $\sigma=1 / 2$ in our simulations.

The second interesting variable is distribution of wealth

$$
D(w)=P\left(w^{\prime}>w\right) .
$$

$P$ means probability that a randomly chosen agent's wealth is greater than $w$.

The following variable is the correlation between wealth and connectivity

$$
H(c)=\bar{w}_{P(w \mid c)} \cdot
$$

Value $H(c)$ is computed as

$$
H(c)=\frac{\sum_{k_{i}=c} w_{i}}{\sum_{k_{i}=c} 1},
$$

where $k_{i}$ is connectivity of individual agent $i$ and $c$ is an integer value.

\section{Results of simulations}

The model was investigated with fixed interaction parameters that were set up to fulfill equation 10 from [37]

$$
2 \beta=(\alpha-1) \varepsilon^{2} \text {. }
$$

with $\alpha=3 / 2$, i.e., the same interaction where the power-law exponent of wealth distribution of the model on the fully-connected network was $\alpha=3 / 2$. Now there is only one freedom, which will be fixed by setting up $\varepsilon=0.01$.

The simulations were performed with the following parameters:

\section{General parameters of the Monte Carlo method}

- Number of agents $N=10000$

- Final time of the simulations $T=1.510^{9}$

- Number of Monte Carlo runs $R=10$

Parameters of the interaction

- $\beta=2.510^{-5}$

- $\varepsilon=0.01$

\section{Parameters of the network}

There are two parameters in the construction of the small-world network using the Watts-Strogatz algorithm [39]:

- Initial number of edges from agent $m=4$ (mean connectivity)

- Probability $p \in[0,1]$ of rewiring of the edge.

The initial wealth of the agents was set at 1 , so the initial wealth dispersed in the society of $N$ agents is $N$.

\subsection{Agent initiated model}

The model is based on random choice of agents that will interact using motion equation 1 . The time evolution of social tension (Fig. 1) for all $p$ rises and then decreases, but in the case of parameter $p<p_{a}, 0.00007<p_{a}<0.0001$, the process is slower and for the subset of cases with $p \neq 0$ there is a trough or plateau in the time evolution after the peak of social tension. The case with $p>p_{a}$ behaves differently: there is one peak and then a rapid decrease in social tension.

The distribution of wealth (Fig. 2) permits the power-law tail for $p>p_{a}$ (the same symbol is used as a consequence of the power-law behavior and the different social tension evolution) with exponent -0.96 , which is stable for the interval of $p$ at the thermodynamic limit $N \rightarrow+\infty, T \rightarrow+\infty$ and $N / T$ constant. The power-law is valid for approximately $1-5 \%$ of the population, which is in quite good agreement with the mea- 


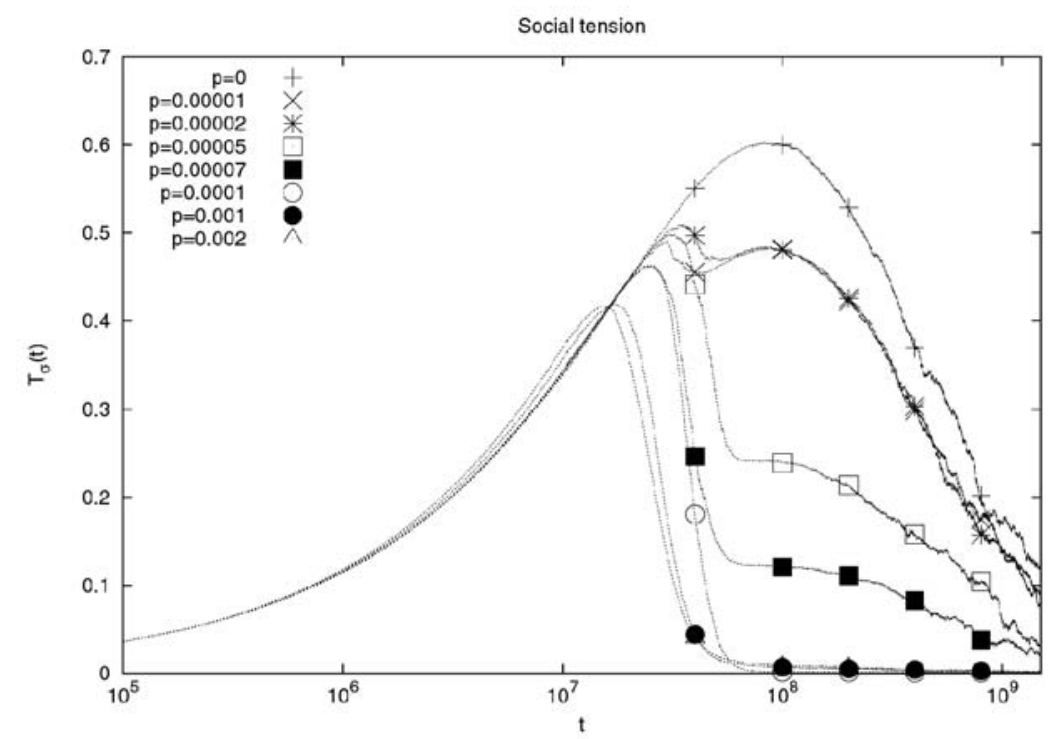

Fig. 1: Time evolution of social tension in the agent initiated model

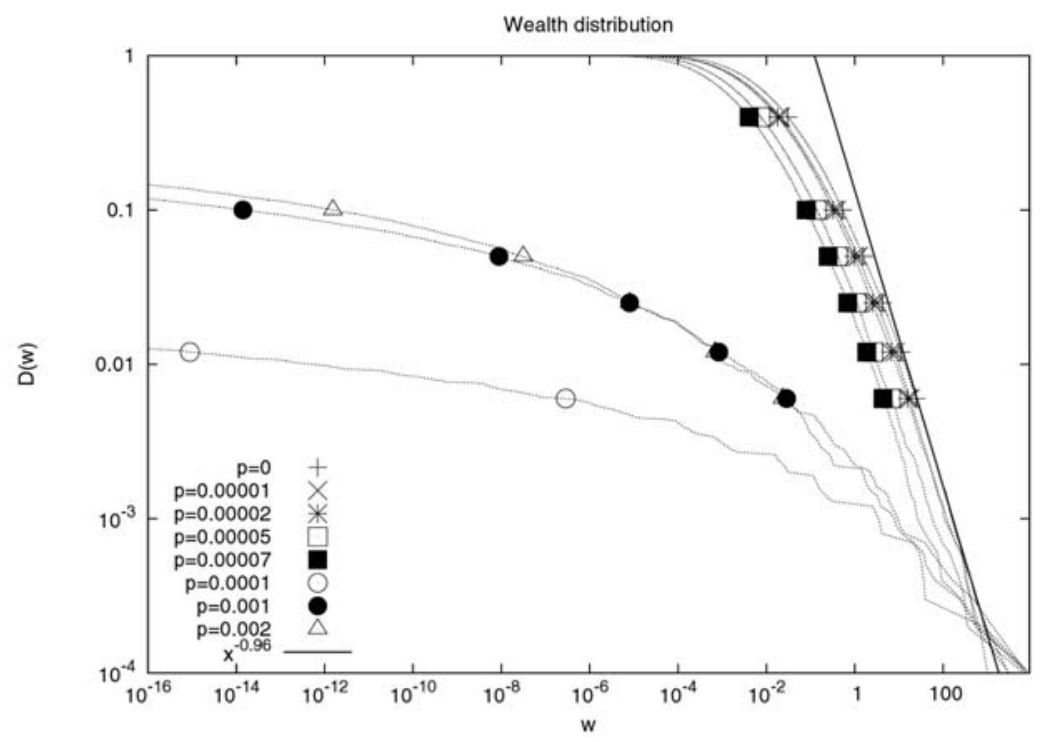

Fig. 2: Distribution of wealth among agents in the agent initiated model

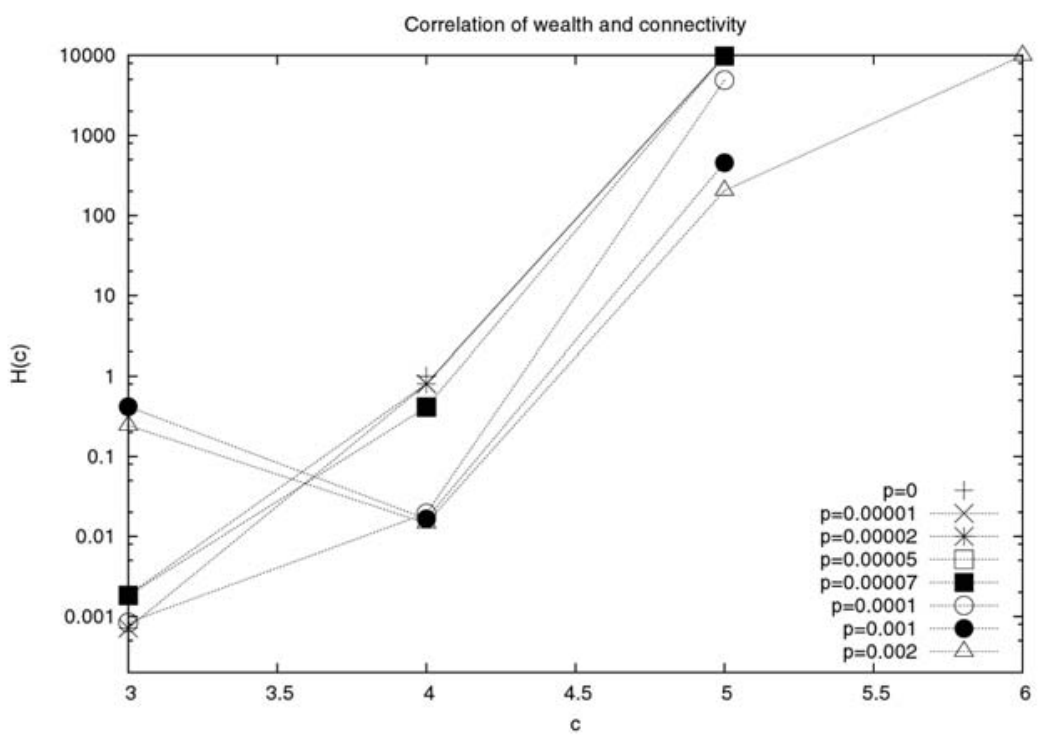

Fig. 3: Correlation of wealth and connectivity in the agent initiated model 
surements in [25]. The deviation of the data from the power-law for the higher end of distribution behaves as a finite-size effect. If $p>p_{a}$, behavior of wealth distribution is no longer power-law, and the initial power-law tail is spread by the dynamics of the model.

In Fig. 3, the average connectivity of the network was 4, and the connectivity is dispersed around this value during the rewiring process. In the case $p<p_{c}$, there is a strong correlation between average wealth and connectivity, but the case $p>p_{a}$ enables less connected agents to outperform agents with average connectivity.

\subsection{Edge initiated model}

The model is based on a random choice of edges that will interact using motion equation 1. Time evolution of social tension (Fig. 4) seems very similar to the previous case. In the case of $p<p_{e}, 0.00007<p_{e}<0.0001$, the dynamic is slower than in the following case, and for $p>0$ there is a peak and a plateau, or a twin peak. This is in contrast to the case $p>p_{e}$, where there is only one peak and then a rapid decrease.
The distribution of wealth (Fig. 5) allows power-law behavior with exponent $\alpha=-0.95$ for the case $p<p_{e}$ and the power-law tail is stable at the thermodynamic limit. As in the previous case it is valid for $1-5 \%$ of the population and the deviation from the power-law for the higher end of the distribution is a finite-size effect. However, there is no power-law for $p>p_{e}$.

The correlation of wealth (Fig. 6) shows that average wealth is a strictly growing function of connectivity $c$. The average wealth of a player with average connectivity (4) is better for $p<p_{e}$, which is similar to the previous case.

\section{Conclusions}

A model of wealth distribution based on inelastical scattering interaction was simulated on the Watts-Strogatz network with the aim of obtaining the powerlaw tail in the higher end of the distribution, which corresponds with Pareto's empirical observations. There are intervals $p$ where the model admits the power-law $p<p_{g}, g \in\{a, e\}$, which is stable at the thermo-

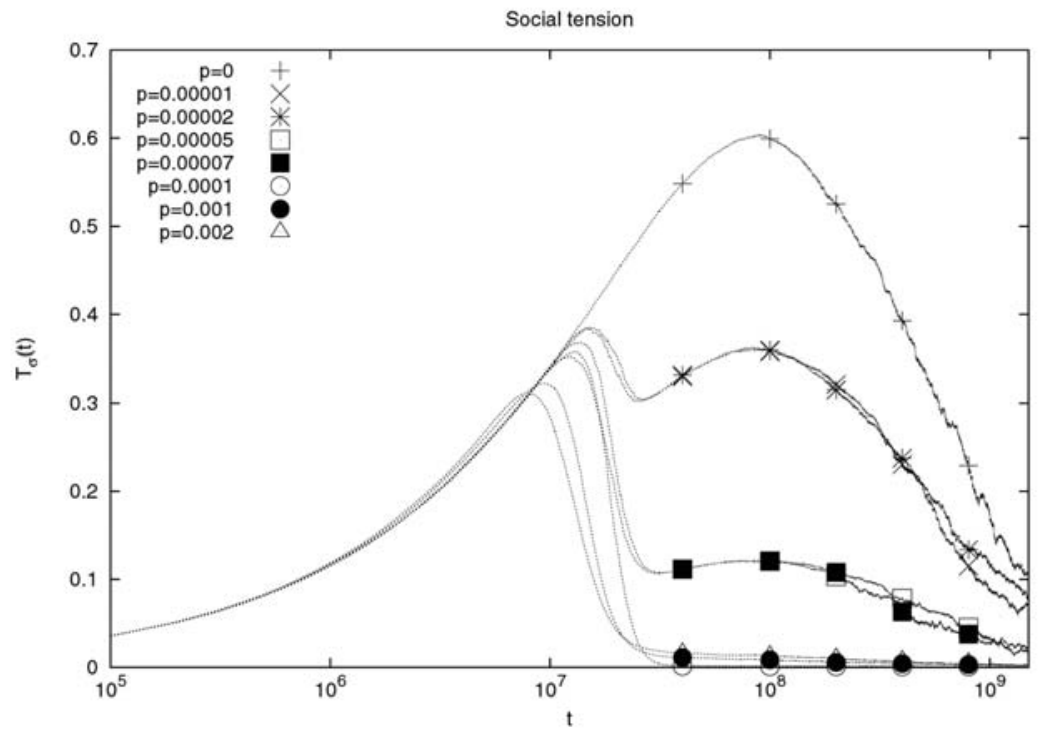

Fig. 4: Time evolution of social tension in the edge initiated model

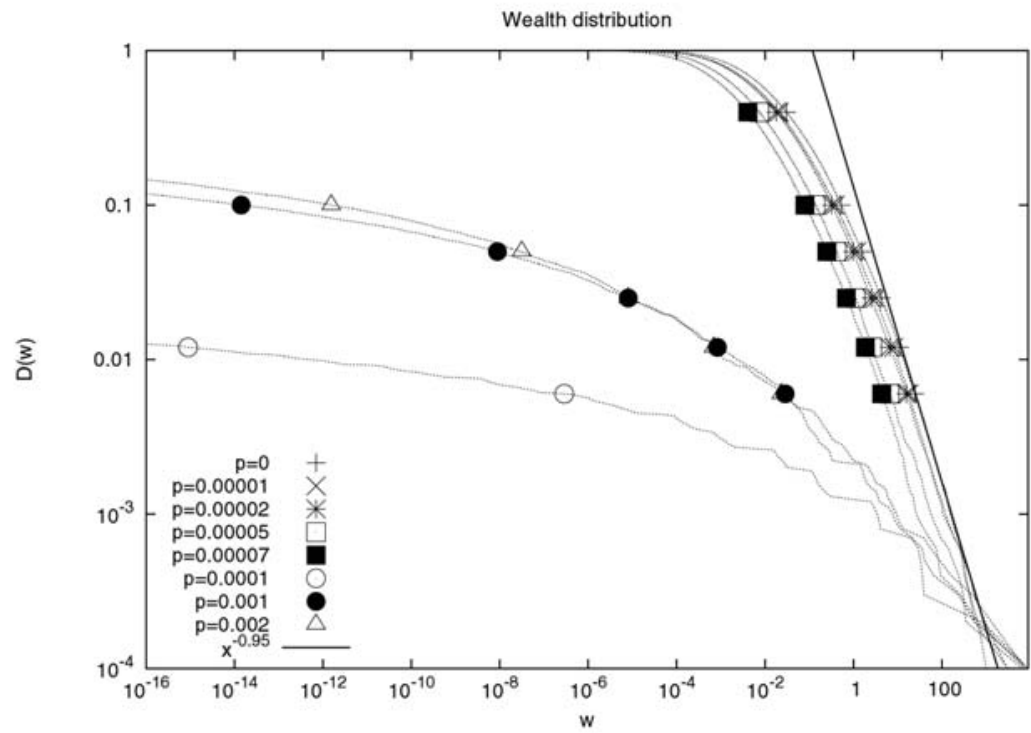

Fig. 5: Distribution of wealth among agents in the edge initiated model 


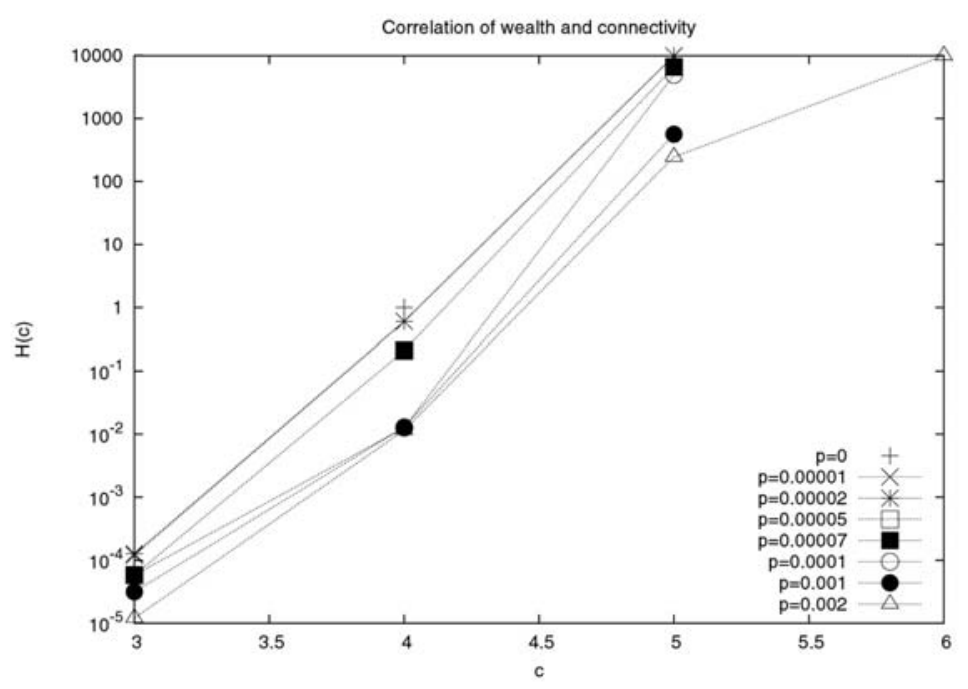

Fig. 6: Correlation of wealth and connectivity in the agent initiated model

dynamic limit and $p>p_{g}$ where there is no longer the power-law. So the model admits the power-law only for a "closed" community without many merchants that trade with distant communities. The exponents, which were measured in the simulations, differ from the mean-field computations in [37] with the exponent $\alpha=3 / 2$.

Connectivity of the agents was a positive factor for wealth, although there are counter-cases. This is especially true for higher connectivities.

\section{Acknowledgments}

The computer simulations in this paper were made on a cluster that is supported by the Department of Physics of the Faculty of Nuclear Sciences and Physical Engineering, CTU in Prague. This work was supported by grant FRVS 2005:3305010.

\section{References}

[1] Pareto, V.: Cours d'economie politique. Lausanne: F. Rouge, 1897.

[2] Levy, M., Solomon, S.: Physica. A 242, 90 (1997).

[3] Dragulescu, A., Yakovenko, V. M.: Physica. A 299, 213 (2001).

[4] Reed, W. J., Hughes, B. D.: Phys. Rev. E 66, 067103 (2002).

[5] Aoyama, H., Souma, W., Fujiwara, Y.: Physica. A 324, 352 (2003).

[6] Abul-Magd, A. Y.: Phys. Rev. E 66, 057104 (2002).

[7] Levy, M., Solomon, S.: Int.J. Mod. Phys. C 7, 595 (1996).

[8] Levy, M., Solomon, S.: Int. J. Mod. Phys. C 7, 65 (1996).

[9] Biham, O., Malcai, O., Levy, M., Solomon, S.: Phys. Rev. E 58, 1352 (1998).

[10] Solomon, S.: "In Decision Technologies for Computational Finance.” Ed. A.-P. Refenes, A. N.Burgess, J. E. Moody, Kluwer Academic Publishers, 1998.
[11] Solomon, S.: "In Application of Simulation to Social Sciences.” Ed. G. Ballot, G. Weisbuch, Hermes Science Publications, 2000.

[12] Huang, Z.-F., Solomon, S.: Eur. Phys.J. B 20, 601 (2001).

[13] Solomon, S., Richmond, P.: Physica. A 299, 188 (2001).

[14] Blank, A., Solomon, S.: Physica. A 287, 279 (2000).

[15] Solomon, S., Levy, M.: Cond-Mat/0005416.

[16] Huang, Z.-F., Solomon, S.: Physica. A 294, 503 (2001).

[17] Sornette, D., Cont, R.: J. Phys. I France 7, 431 (1997).

[18] Sornette, D.: Physica A 250, 295 (1998).

[19] Sornette, D.: Phys. Rev. E 57, 4811 (1998).

[20] Takayasu, H., Sato, A. -H., Takayasu, M.: Phys. Rev. Lett. 79, 966 (1997).

[21] Marsili, M., Maslov, S., Zhang, Y. -C.: Physica. A 253, 403 (1998).

[22] Bouchaud, J. -P., Mézard, M.: Physica. A 282, 536 (2000).

[23] Burda, Z., Johnson, D., Jurkiewicz, J., Kaminski, M., Nowak, M. A., Papp, G., Zahed, I.: Cond-Mat/0101068.

[24] Dragulescu, A., Yakovenko, V. M.: Eur. Phys.J. B 17, 723 (2000); Eur. Phys. J. B 20, 585 (2001); in "Modeling of Complex Systems: Seventh Granada Lectures", AIP Conference Proceedings 661, 180 New York, 2003.

[25] Yakovenko, V. M.: Cond-Mat/0302270.

[26] Ispolatov, S., Krapivsky, P. L., Redner, S.: Eur. Phys. J. B 2, 267 (1998).

[27] Chakraborti, A., Chakrabarti, B. K.: Eur. Phys. J. B 17, 167 (2000).

[28] Chakrabarti, B. K., Chatterjee, A.: “Applications of Econophysics." Conference proceedings of second Nikkei Symposium on Econophysics, Tokyo, Japan, 2002, Springer-Verlag, 2003.

[29] Chatterjee, A., Chakrabarti, B. K., Manna, S. S.: Physica. A 335, 155-163 (2004); Phys. Scripta. T 106, 36-38 (2003).

[30] Scafetta, N., Picozzi, S., West, B. J.: Cond-Mat/0209373.

[31] Scafetta, N., West, B. J.: “A Trade-Investment Model for Distribution of Wealth, Nonlinear Dynamics and Nonextensivity." Conference proceedings of Workshop on 
Anomalous Distributions, Nonlinear Dynamics and Nonextensivity, Santa Fe, USA.

[32] Gligor, M., Ignet, M.: Eur. Phys. J. B 30, 125 (2002).

[33] Sinha, S.: Physica Scripta. T 106, 59-64 (2003).

[34] Jaeger, H. M., Nagel, S. R., Behringer, R. P.: Rev. Mod. Phys. 68, 1259 (1996).

[35] Bobylev, A. V., Carillo, J. A., Gamba, I. M.: J. Stat. Phys. 98, 743 (2000).

[36] Bobylev, A. V., Cercignani, C.: J. Stat. Phys. 106, 547 (2002); J. Stat. Phys. 110, 333 (2003).

[37] Slanina, F.: Phys. Rev. E 69, 046102 (2004).

[38] Albert, R., Barabási, A. -L.: Reviews of Modern Physics. 74, 47 (2002).

[39] Watts, D. J., Strogatz, S. H.: Nature. 393, 440 (1998).

[40] Barabási, A. -L., Albert, R.: Science. 286, 509 (1999).
Ing. Hynek Lavička

e-mail: lavicka@fjfi.cvut.cz

Department of Physics

Czech Technical University in Prague

Faculty of Nuclear Sciences and Physical Engineering Břehová 7

11519 Praha 1, Czech Republic

RNDr. František Slanina, CSc.

tel. +420266052671

slanina@fzu.cz

Institute of Physics

Academy of Sciences of the Czech Republic

Na Slovance 2

18221 Praha 8, Czech Republic 